\title{
Características epidemiológicas de pacientes portadores de neoplasias de cabeça e pescoço submetidos à radioterapia em Juiz de Fora - MG
}

\author{
Bruna Quintão Costa Rocha* \\ Luis Eneas* \\ Rodrigo Guerra de Oliveira* \\ Francielle Silvestre Verner** \\ Rafael Binato Junqueira**
}

\begin{abstract}
RESUMO
A neoplasia de cabeça e pescoço é o sexto tipo de câncer mais comum no mundo. As neoplasias malignas maxilofaciais, em sua maioria, se iniciam nas células escamosas que revestem as superfícies mucosas da região. O objetivo no presente estudo foi realizar um levantamento epidemiológico sobre neoplasias de cabeça e pescoço em pacientes que se submeteram à radioterapia em um hospital de alta complexidade oncológica da cidade de Juiz de Fora, Minas Gerais (MG). Foram incluídos na análise prontuários de pacientes, de ambos os sexos, de todas as faixas etárias e etnias, com diagnóstico de neoplasias de cabeça e pescoço e que estiveram sob tratamento de radioterapia no período de janeiro a setembro de 2015, no Hospital ASCOMCER, situado em Juiz de Fora (MG). A idade dos pacientes acometidos com neoplasias de cabeça e pescoço variou de 39 a 88 anos, com idade média de 59,78 $( \pm 12,49)$ anos, e faixa etária de 61 a 70 anos apresentando prevalência das alterações. Em relação ao sexo, observou-se uma maior prevalência das lesões neoplásicas em homens (64,3\%) do que em mulheres (35,7\%). Em relação ao sitio anatômico primário, a laringe foi o local mais prevalente $(17,9 \%)$ e o lábio inferior (3,6\%), assoalho bucal $(3,6 \%)$ e glote $(3,6 \%)$ os menos prevalentes. Observou-se um maior número de pacientes sem comprometimento dos linfonodos regionais $(53,6 \%)$, do que pacientes com comprometimento (46,4\%). Este estudo forneceu o perfil epidemiológico dos pacientes portadores de neoplasias de cabeça e pescoço, e que necessitaram de tratamento com radioterapia, no referido hospital. Tal delineamento epidemiológico é fundamental para auxiliar no planejamento de ações de prevenção futuras na cidade de Juiz de Fora.
\end{abstract}

Palavras-chave: Epidemiologia. Neoplasias. Radioterapia.

\section{INTRODUÇÃO}

A neoplasia de cabeça e pescoço é o sexto tipo de câncer mais comum no mundo. As neoplasias malignas maxilofaciais, em sua maioria, se iniciam nas células escamosas que revestem as superfícies mucosas da região. Tais condições podem acarretar disfunções psicossociais na vida diária de um indivíduo, gerando também repercussões psicológicas em pessoas de seu convívio. O alto consumo de álcool e tabaco é apontado como uma das principais causas de aumento dessa doença nas últimas décadas. Os principais sinais e sintomas que podem ser apresentados pelos pacientes são: dor, disfagia, nódulo cervical, sangramento e dificuldades na abertura bucal (MACHIELS et al., 2014; INCA, 2015).

O tempo decorrido entre os primeiros sinais e sintomas, diagnóstico e tratamento interferem na evolução, prognóstico e qualidade de vida do paciente portador da doença. O atraso no diagnóstico da neoplasia de cabeça e pescoço é a principal causa de um prognóstico ruim. Ressalta-se que uma possível cura depende exclusivamente do diagnóstico precoce e de uma maior ênfase na conscientização do profissional cirurgião-dentista e do próprio paciente (SANTOS, BATISTA, CANGUSSU, 2010). O tratamento da neoplasia de boca e orofaringe pode ser cirúrgico, radioterápico, quimioterápico ou combinações entre estes métodos. Quando uma lesão maligna é diagnosticada em sua fase inicial, ou seja, menor que $2 \mathrm{~cm}$ de extensão e sem comprometimento linfonodal (T1, N0, M0), o tratamento cirúrgico local será provavelmente suficiente para curar o paciente. Entretanto, se o diagnóstico não é feito no início, a extensão local certamente ocorrerá, podendo haver invasão dos linfonodos regionais. Isto altera o estadiamento da neoplasia (T2, T3, T4) e o

\footnotetext{
* Hospital Maternidade Therezinha de Jesus-HMTJ/JF e Suprema-Faculdade Ciências Médicas e da Saúde Juiz de Fora - Juiz de Fora, MG.

** Universidade Federal de Juiz de Fora, Campus Avançado Governador Valadares, Departamento de Odontologia - Governador Valadares, MG.
} 
tratamento deverá incluir radioterapia e, em alguns casos, quimioterapia (SANTOS et al., 2012).

A radioterapia, juntamente com a quimioterapia e cirurgia são as formas terapêuticas utilizadas para o tratamento de neoplasias malignas de cabeça e pescoço. Apesar dos benefícios apresentados pela radioterapia, esse método apresenta alguns efeitos colaterais que, em grande parte das vezes, se manifestam na cavidade oral como xerostomia, dermatite, mucosite, candidíase, alterações do paladar, disfagia, lesões cariosas, trismo e osteorradionecrose (MACHIELS et al., 2014).

Mediante o exposto, o objetivo no presente estudo foi realizar um levantamento epidemiológico sobre neoplasias de cabeça e pescoço em pacientes que se submeteram à radioterapia em um hospital de alta complexidade oncológica da cidade de Juiz de Fora, Minas Gerais (MG), no ano de 2015.

\section{Material e Métodos}

Este estudo, do tipo retrospectivo observacional transversal, foi submetido e aprovado pelo Comitê de Ética em Pesquisa com Seres Humanos, da Faculdade de Ciências Médicas e da Saúde de Juiz de Fora (1.245.013/2015).

Foram incluídos na análise prontuários de pacientes, de ambos os sexos, de todas as faixas etárias e etnias, com diagnóstico de neoplasias de cabeça e pescoço e que estiveram sob tratamento de radioterapia no período de janeiro a setembro 2015, no Hospital ASCOMCER, situado em Juiz de Fora, MG. Foram excluídos do estudo pacientes que não possuíam diagnóstico histopatológico confirmado ou que apresentavam neoplasias em outras áreas do corpo.

Após a aplicação dos critérios de inclusão e exclusão, foram analisados 28 prontuários quanto à idade, sexo, sítio anatômico primário das neoplasias de cabeça e pescoço e comprometimento de linfonodos regionais.

Os dados coletados foram reunidos em um banco de dados elaborado exclusivamente para este estudo, e analisados por estatística descritiva por meio do software SPSS versão 21.0.

\section{Resultados}

A idade dos pacientes acometidos com neoplasias de cabeça e pescoço variou de 39 a 88 anos, sendo a idade média de 59,78 $( \pm 12,49)$ anos. A distribuição dos pacientes por faixa etária encontra-se na Tabela 1.

\section{TABela 1}

Faixa etária dos pacientes com câncer e cabeça e pescoço

\begin{tabular}{lc}
\hline & $\mathrm{N}^{\text {o }}$ de pacientes $(\%)$ \\
\hline $39-50$ anos & $6(21,4)$ \\
$51-60$ anos & $8(28,6)$ \\
$61-70$ anos & $10(35,7)$ \\
$71-80$ anos & $1(3,6)$ \\
$81-90$ anos & $3(10,7)$ \\
TOTAL: & $28(100)$ \\
\hline
\end{tabular}

Fonte: Os autores (2016).

A distribuição da prevalência das lesões em relação ao sexo encontra-se na Tabela 2.

\section{TABela 2}

Sexo dos pacientes com câncer e cabeça e pescoço

\begin{tabular}{lc}
\hline & $\mathrm{N}^{\circ}$ de pacientes $(\%)$ \\
\hline Mulheres & $10(35,7)$ \\
Homens & $18(64,3)$ \\
\hline Fonte: Os autores (2016). &
\end{tabular}

Em relação ao sitio anatômico primário, foram encontradas lesões de origem no lábio inferior, base da língua, assoalho bucal, palato mole, orofaringe, nasofaringe, laringe, glândula parótida, glândula tireóide, glote e sítio anatômico desconhecido. A laringe foi o sitio mais acometido com $17,9 \%$, seguida dos tumores de base da língua $(14,3 \%)$ e tumores com sítios primários desconhecidos (14,3\%). E as regiões menos acometidas foram assoalho bucal, glote e o lábio inferior, com 3,6\% cada. A distribuição dos casos de acordo com o sítio anatômico acometido encontra-se na Tabela 3.

\section{TABELA 3}

Sítio anatômico primário dos pacientes com câncer de cabeça e

\begin{tabular}{lc}
\multicolumn{2}{c}{ Pescoç0 } \\
\hline Lábio Inferior & $\mathrm{N}^{\circ}$ de pacientes (\%) \\
Base da Língua & $1(3,6)$ \\
Assoalho bucal & $4(14,3)$ \\
Palato Mole & $1(3,6)$ \\
Orofaringe & $3(10,7)$ \\
Nasofaringe & $2(7,1)$ \\
Laringe & $2(7,1)$ \\
Glândula Parótida & $5(17,9)$ \\
Glândula Tireoide & $3(10,7)$ \\
Glote & $2(7,1)$ \\
Desconhecido & $1(3,6)$ \\
Fonte: Os autores (2016). & $4(14,3)$ \\
\hline
\end{tabular}

A frequência de comprometimento dos linfonodos regionais encontra-se na tabela 4 . 


\section{TABELA 4}

Frequência de comprometimento de linfonodos regionais

\begin{tabular}{lc}
\hline & $\mathrm{N}^{\circ}$ de pacientes $(\%)$ \\
\hline Ausência de comprometimento & $15(53,6)$ \\
Comprometimento & $13(46,4)$ \\
\hline Fonte: Os autores (2016).
\end{tabular}

\section{Discussão}

A neoplasia de cabeça e pescoço pode acarretar disfunções psicossociais na vida diária de um indivíduo. A etiologia do câncer está relacionada à interação entre fatores endógenos - genéticos e ambientais, sendo que sua grande maioria está associada a fatores ambientais, sendo eles: exposição à radiação, o uso de tabaco, a ingestão de álcool, a obesidade, o sedentarismo e o consumo de nitritos e nitratos, destacando-se o tabagismo e a ingestão de álcool. O fator genético exerce um papel importante na oncogênese, porém são de menor incidência os casos exclusivamente relacionados a fatores hereditários, familiares e étnicos (TOSCANO et al., 2008). O alto consumo de álcool e/ou tabaco é apontado como uma das principais causas de aumento dessa doença (MACHIELS et al., 2014; INCA, 2015), porém estes possíveis fatores etiológicos não estavam disponíveis nos prontuários de todos os pacientes, e não foram avaliados nesta pesquisa.

O presente estudo revelou a predominância de neoplasias de cabeça e pescoço em indivíduos do sexo masculino corroborando com outros achados da literatura que se propuseram a investigar a mesma temática (CASATI et al., 2012). A idade média dos pacientes acometidos com neoplasias de cabeça e pescoço nos prontuários avaliados variou de 39 a 88 anos, sendo a idade média de 59,78, estando de acordo com os achados da literatura (MANFRO et al., 2006).

Lesões iniciais, geralmente assintomáticas, além da carência de conhecimento de diagnóstico diferencial por parte dos profissionais de saúde, são fatores que contribuem para que os pacientes demorem a procurar tratamento, só buscando auxílio em fases tardias do estadiamento da doença, geralmente quando os tumores atingem estágios avançados (T3 e T4), já com indicações de radioterapia e em alguns casos, quimioterapia (SANTOS, BATISTA, CANGUSSU, 2010).

A radioterapia pode ser realizada como uma alternativa ao tratamento cirúrgico e quimioterápico contra o câncer, ou como uma terapia combinada. O tratamento radioterápico tem por função destruir as células neoplásicas para que haja redução ou desaparecimento da neoplasia maligna. Entretanto, as elevadas doses de radiação utilizadas podem gerar efeitos deletérios nas estruturas maxilofaciais, como por exemplo, xerostomia, mucosite, ageusia e cáries de radiação, dentre outras (SAWATA, DIAS, ZAGO, 2006). Quando os pacientes passam por avaliação Odontológica, com tratamento de lesões cariosas e processos inflamatórios (ex. lesões osteolíticas inflamatórias periapicais e lesões periodontais), com consequente adequação do meio bucal previamente ao tratamento radioterápico, as necessidades de tratamentos posteriores diminuem (ROSALES et al., 2010).

O sítio primário mais acometido foi a laringe, se diferenciando da literatura, a qual demonstra predileção pela cavidade oral (ALVARENGA et al., 2008), o que pode ser explicado por hábitos como o tabagismo e o consumo de álcool, aumentando o risco de incidência dessas lesões duas ou três vezes na cavidade oral. Observou-se um maior número de pacientes sem comprometimento dos linfonodos regionais, o que não está de acordo com os resultados de Alvarenga e outros (2008). Tal fato pode estar relacionado ao número diferente de prontuários incluídos no presente estudo e no estudo citado anteriormente.

O prognóstico se diferencia em cada localização do sítio anatômico primário, no tamanho do tumor, na presença de metástase em linfonodos cervicais e de metástase à distância. Porém, acredita-se que a presença de metástase em linfonodos cervicais seja o principal fator prognóstico independente, uma vez que em muitos casos, devido à ausência de sinais $\mathrm{e}$ sintomas nos estágios iniciais, o paciente apenas procura ajuda profissional em estágios avançados da doença (T3 e T4), já havendo comprometimento dos linfonodos cervicais, tornando o prognóstico duvidoso (ALVARENGA et al., 2008; COLOMBO, RAHAL, 2009; ALMEIDA et al., 2011).

Apesar da relevância clínica do presente estudo, sugere-se a realização de novas pesquisas que avaliem os principais efeitos deletérios da radiação no complexo bucomaxilofacial em pacientes submetidos à radioterapia para tratamento de neoplasias de cabeça e pescoço. A busca de métodos que amenizem ou eliminem tais efeitos, poderá representar uma forma de melhor a qualidade de vida deste grupo vulnerável.

\section{Conclusão}

A neoplasia de cabeça e pescoço foi mais prevalente em indivíduos do sexo masculino, na faixa etária de 61 a 70 anos, com maior acometimento na laringe e sem comprometimento dos linfonodos.

Este estudo forneceu o perfil epidemiológico dos pacientes portadores de neoplasias de cabeça e pescoço, e que necessitaram de tratamento com

HU Revista, Juiz de Fora, v. 43, n. 1, p. 71-75, jan./jun. 2017 
radioterapia, no Hospital ASCOMCER, no ano de 2015. Tal delineamento epidemiológico é fundamental para auxiliar no planejamento de ações de prevenção futuras na cidade de Juiz de Fora.
Cabe ao cirurgião dentista contribuir no diagnóstico precoce e diferencial destes pacientes, já que quanto mais tardio o diagnóstico pior são as taxas de morbidade e mortalidade neste grupo de pacientes.

\title{
Epidemiological aspects of patients with head and neck neoplasms undergoing radiotherapy in Juiz de Fora - MG
}

\begin{abstract}
Head and neck neoplasm is the sixth most common type of neoplasia worldwide. The maxillofacial malignancies mostly begin in the squamous cells that line the moist surfaces of the region. The aim of the present study was to conduct an epidemiological survey of head and neck neoplasms in patients who underwent radiotherapy in a hospital with high oncological complexity in the city of Juiz de Fora, Minas Gerais, Brazil. The analysis included records of patients of both sexes, of all ages and ethnicities, diagnosed with head and neck neoplasm and who are underwent radiation therapy treatment from January to September 2015, at ASCOMCER Hospital (Juiz de Fora, MG, Brazil). The age of affected patients with head and neck tumors ranged from 39 to 88 years, with a mean age of $59.78( \pm 12.49)$ years, with the lesions being prevalent in the age group 61-70 years. In relation to sex, it was observed a higher prevalence of neoplastic lesions in men $(63.3 \%)$ than in women (35.7\%). For the primary anatomical site, the larynx was the most prevalent site (17.9\%), and lower lip (3.6\%), buccal floor $(3.6 \%)$ and glottis $(3.6 \%)$ were the lowest. A larger number of patients without regional lymph node involvement $(53.6 \%)$ can be observed than patients with involvement (46.4\%). This study provided the epidemiological profile of patients with head and neck neoplasms who underwent treatment with radiotherapy at the ASCOMCER Hospital in the year 2015. Such an epidemiological delineation is fundamental to assist in the planning of future prevention actions in the city of Juiz de Fora.
\end{abstract}

Key words: Epidemiology. Neoplasms. Radiotherapy.

\section{REFERENCIAS}

ALMEIDA, F. C. S. et al. Fatores prognósticos no câncer de boca. Revista Brasileira de Ciências da Saúde, João Pessoa, v. 15, n. 4, p. 471-8, out/dez., 2011.

ALVARENGA, L. M. et al. Avaliação epidemiológica de pacientes com câncer de cabeça e pescoço em um hospital universitário do noroeste do estado de São Paulo. Revista Brasileira de Otorrinolaringologia, São Paulo, v. 74, n. 1, p. 68-73, jan/fev. 2008.

CARDOSO, M. F. A. et al. Prevenção e controle das sequelas bucais em pacientes irradiados por tumores de cabeça e pescoço. Radiologia Brasileira, São Paulo, v. 38, n. 2, p. 107-15, mar/abr. 2005.

CASATI, M. F. M. et al. Epidemiologia do câncer de cabeça e pescoço no Brasil: estudo transversal de base populacional. Revista Brasileira de Cirurgia de Cabeça e Pescoço, São Paulo, v. 41, n. 4, p. 186-91, out/dez. 2012.

COLOMBO, J.; RAHAL, P. Alterações genéticas em câncer de cabeça e pescoço. Revista Brasileira de Cancerologia, Rio de Janeiro, v. 55, n. 2, p. 165-74, 2009.
INCA. Instituto Nacional do Câncer. Disponível em: http:/ / www2.inca.gov.br/wps/wcm/connect/tiposdecancer/site/ home/boca. Acesso em 08 de dez. 2015.

MACHIELS, J. P. et al. Advances in the management of squamous cell carcinoma of the head and neck. F1000Prime Reports, v. 2, n. 6, p. 1-10, jun. 2014.

MANFRO, G. et al. Relação entre idade, sexo, tratamento realizado e estágio da doença com a sobrevida em pacientes terminais com carcinoma epidermoide de laringe. Revista Brasileira de Cancerologia, Rio de Janeiro, v. 52, n. 1, p. 17-24, 2006.

ROSALES, A. C. M. N. et al. Dental needs in brazilian patients subjected to head and neck radiotherapy. Brazilian Dental Journal, Ribeirão Preto, v. 20, n. 1, p. 74-7, 2009.

SANTOS, L. C. O.; BATISTA, O. M.; CANGUSSU, M. C. T. Characterization of oral cancer diagnostic delay in the state of Alagoas. Brazilian Journal of Otorhinolaryngoly, São Paulo, v. 76, n. 4, p. 416-22, jul/ago. 2010. 
SANTOS, V. C. B. et al. Câncer de boca: análise do tempo decorrido da detecção ao início do tratamento em centro de Oncologia de Maceió. Revista Brasileira de Odontologia, Rio de Janeiro, v. 69, n. 2, p. 159-64, jul/dez. 2012.
SAWATA, N. O.; DIAS, A.M.; ZAGO, M. M. F. Efeito da radioterapia sobre a qualidade de vida dos pacientes com câncer de cabeça e pescoço. Revista Brasileira de Cancerologia, Rio de Janeiro, v. 52, n. 4, p. 323-29, out/dez. 2006.

TOSCANO, B. A. F. et al. Câncer: implicações nutricionais.

Comunicação em Ciências da Saúde, v. 19, n. 2, p. 171-80, 2008.

Enviado em 04/10/2016 